\title{
Variabilidad del peso en mujeres con cáncer de mama (IIIB) después de tratamiento
}

\author{
Weight change in women with breast cancer (IIIB) \\ after treatment
}

\author{
Guillermo Andrés Herrera-Rueda, Jesús Solier Insuasty-Enríquez, \\ Reynaldo Mauricio Rodríguez-Amaya, Nathalia Andrea Martínez-Moreno, \\ José Fernando Lozano-Jiménez, Daniela Reyes-Cadena, Jonnathan Rueda-Lara \\ - Bucaramanga (Colombia)
}

DOI: https://doi.org/10.36104/amc.2020.1563

\section{Resumen}

Introducción: se ha descrito ampliamente el efecto del sobrepeso y la obesidad en pacientes con cáncer de mama. A pesar de que se reconocen diferencias étnicas de dichas asociaciones, aún es insuficiente la información en población latinoamericana.

Metodología: estudio longitudinal tipo cohorte retrospectiva, con muestreo no probabilístico. El objetivo principal fue describir el comportamiento del peso de mujeres con cáncer de mama, localmente avanzado del subtipo luminal A después de tratamiento oncológico multimodal.

Resultados: la edad promedio al diagnóstico de cáncer fue de 52 años. El tiempo promedio de seguimiento de 2.3 años en los cuales hubo una tasa de recurrencia de $12.1 \%$. La mayoría de las pacientes presentaron sobrepeso/obesidad (67.56\%) con una variación promedio de $-0.17 \mathrm{Kg}$ al final del seguimiento. Las pacientes con metástasis presentaron una pérdida mayor de peso que su contraparte sin recurrencia $(-5.06 \mathrm{Kg}, \mathrm{p}<0.05)$.

Conclusiones: el sobrepeso y obesidad son una característica muy prevalente en pacientes con cáncer de mama luminal A en estadio localmente avanzado. No hubo evidencia concluyente de aumento de riesgo de metástasis o muerte asociado a exceso de peso en esta población. Al contrario la pérdida de peso fue una característica estadísticamente significativa de pacientes que presentaron recurrencia a distancia durante el seguimiento, sin poderse definir como un factor causal. (Acta Med Colomb 2020; 45. DOI: https://doi.org/10.36104/amc.2020.1563).

Palabras clave: peso, sobrepeso, obesidad, cáncer de mamá, metástasis, neoplasia .

\section{Abstract}

Introduction: the effect of overweight and obesity in patients with breast cancer has been widely described. Despite the recognition of ethnic differences in these associations, information is still lacking for the Latin American population.

Methods: a retrospective, longitudinal cohort study with non-probabilistic sampling. The main objective was to describe how weight behaved after multimodal cancer treatment in women with locally advanced luminal A subtype breast cancer.

Results: the average age at cancer diagnosis was 52 years. The average follow-up time was 2.3 years, during which there was a $12.1 \%$ rate of recurrence. Most patients were overweight/obese $(67.56 \%)$, with an average variation of $-0.17 \mathrm{~kg}$ at the end of follow up. Patients with metastasis had a greater weight loss than those without recurrence $(-5.06 \mathrm{~kg}, \mathrm{p}<0.05)$.

Conclusions: overweight and obesity are a prevalent characteristic of locally advanced luminal A breast cancer patients. There was no conclusive evidence of increased risk of metastasis or death related to excess weight in this population. To the contrary, weight loss was a statistically significant characteristic of patients with distal recurrence during follow up, although it was not established as a causal factor. (Acta Med Colomb 2020; 45. DOI: https://doi.org/10.36104/amc.2020.1563).

Key words: weight, overweight, obesity, breast cancer, metastasis, neoplasia.
Dr. Guillermo Andrés Herrera-Rueda: Internista, Investigador Principal; Dr. Jesús Solier Insuasty-Enríquez: Internista, Oncólogo Clínico, Director del Proyecto; Dr. Reynaldo Mauricio Rodríguez-Amaya: Epidemiólogo, Codirector y Asesor Epidemiológico; Dra. Nathalia Andrea Martínez-Moreno: Residente Pediatría, Investigadora Universidad de Antioquia; José Fernando Lozano-Jiménez, Daniela ReyesCadena, Jonnathan Rueda-Lara: Estudiantes de VI Año de Medicina, Grupo de Investigación GERMINA. Universidad Industrial de Santander. Bucaramanga (Colombia). Correspondencia: Dr. Guillermo Andrés Herrera-Rueda. Bucaramanga (Colombia). E-mail: memohrueda_19@hotmail.com Recibido: 07/XI/2019 Aceptado 15/IV/2020 


\section{Introducción}

El sobrepeso es reconocido como uno de los principales factores de riesgo prevenibles de cáncer. Hasta un 30\% de la mortalidad por esta enfermedad podría evitarse si se interviniera el excesivo aumento de peso. Lamentablemente este fenómeno tiene un comportamiento epidémico con una prevalencia en ascenso especialmente en países en vía de desarrollo.

Uno de los prototipos del impacto del sobrepeso en la enfermedad maligna es el cáncer de mama. Esta asociación puede deberse a mecanismos como el aumento del estrés oxidativo, conversión periférica de hormonas sexuales (1), adipocinas y citoquinas inflamatorias involucradas en la inhibición de la apoptosis celular, migración y aumento de la proliferación de células malignas (2).

Desde el punto de vista clínico, varios estudios de cohortes han investigado la asociación entre el sobrepeso/obesidad y el pronóstico del cáncer de mama. La mayoría han reportado una preocupante asociación entre el sobrepeso con reducción de la supervivencia y aumento de la recurrencia a distancia (3-5). Llamativamente un IMC bajo $\left(<18.5 \mathrm{Kg} / \mathrm{m}^{2}\right)$ también mostró reducción en la supervivencia general y específica por cáncer de mama (6).

Teniendo en cuenta que el comportamiento del peso (aumento o descenso) y el cáncer, son fenómenos en salud determinados por factores genéticos y ambientales, es pertinente y se justifica investigar su relación en diferentes poblaciones y territorios. En especial cuando la mayoría del cuerpo de evidencia proviene de mujeres blancas o asiáticas y aún son escasos los resultados en mujeres latinoamericanas.

\section{Metodología}

Se trata de un estudio longitudinal tipo cohorte retrospectiva con objetivos descriptivos que contó con el aval de los comités de ética de las instituciones participantes. Se realizó muestreo no probabilístico por conveniencia con datos provenientes de registros de dos centros de referencia en oncología del nororiente colombiano. El periodo de inclusión de registros médicos va desde 2005 - 2015 (10 años) y se consideraron los siguientes criterios de selección: a) ser mujer mayor de dieciocho años, b) contar con diagnóstico de cáncer de mama invasivo, c) catalogarse en estadio IIIB, d) subtipo luminal A (receptores hormonales positivos y HER2/neu negativo), e) haber recibido tratamiento multimodal (mastectomía, quimioterapia, radioterapia y hormonoterapia con tamoxifeno), f) no tener sospecha clínica de enfermedad residual (local) posoperatoria o recurrencia al principio del seguimiento (primer año) y f) tener al menos dos registros de peso con una diferencia mínima de seis meses entre sí, después de iniciada la terapia oncológica.

El objetivo principal fue describir el comportamiento del peso en las pacientes elegidas durante un periodo de 24 meses de seguimiento después de terminado el tratamiento oncológico multimodal. Además, se exploraría la relación con los desenlaces recurrencia a distancia (metástasis) y mortalidad reconociendo los alcances de la muestra disponible.

El análisis de los resultados se realizó valiéndose del paquete estadístico STATA $14.0 ®$ y Microsoft Excel ${ }^{\circledR}$ aplicando medidas de tendencia central, dispersión, comparación de proporciones $\left(\chi^{2}\right)$ y análisis de varianzas. Se consideró la significancia estadística con un valor de $\mathrm{p}<0.05$.

\section{Resultados}

Se revisaron 1662 registros con diagnóstico de cáncer de mama, de éstos cumplieron los estrictos criterios de selección 74 pacientes. Las características sociodemográficas se describen en la Tabla 1.

El seguimiento promedio después de terminado el tratamiento oncológico fue 2.33 años. Se presentaron nueve eventos de recurrencia tumoral, cinco correspondían a metástasis ósea, tres pulmonares y una cerebral, que a su vez se relacionó con la única mortalidad registrada.

Ninguna paciente presentó un IMC $<18 \mathrm{Kg} / \mathrm{m}^{2}$, por el contrario $67.56 \%$ se clasificaron desde el primer registro en sobrepeso/obesidad. Al final del seguimiento el subgrupo en sobrepeso aumentó principalmente por migración de pacientes obesas que perdieron peso. Sin embargo, ninguna

Tabla 1. Características sociodemográficas.

\begin{tabular}{|c|c|}
\hline $\begin{array}{c}\text { Característica } \\
\text { (n) }\end{array}$ & $\begin{array}{c}\text { Porcentaje general } \\
\%\end{array}$ \\
\hline Edad diagnóstico (74) & 52.01 años (SD 10.76, 26-78) \\
\hline $\begin{array}{l}\text { Residencia (72) } \\
\quad \text { Rural } \\
\text { Urbano }\end{array}$ & $\begin{array}{c}\% \\
12.50(9) \\
87.50(63)\end{array}$ \\
\hline $\begin{array}{c}\text { Estrato (48) } \\
\text { Uno } \\
\text { Dos } \\
\text { Tres } \\
\text { Cuatro } \\
\text { Cinco } \\
\text { Seis }\end{array}$ & $\begin{array}{c}\% \\
4.17(2) \\
20.83(10) \\
37.50(18) \\
33.33(16) \\
2.08(1) \\
2.08(1)\end{array}$ \\
\hline $\begin{array}{l}\text { Estado civil (57) } \\
\text { Soltero } \\
\text { Casado } \\
\text { Unión libre }\end{array}$ & $\begin{array}{c}\% \\
33.33(19) \\
8.77(5) \\
57.89(33)\end{array}$ \\
\hline Edad menarca (42) & 13.54 años (SD 1.64, 10-18) \\
\hline Edad menopausia (9) & 48.55 años (SD 4.69, 41-55) \\
\hline Embarazos a término (70) & 2.97 (DE 2.28, 0-14) \\
\hline $\begin{array}{l}\text { Lactancia (30) } \\
\text { No } \\
\text { Si }\end{array}$ & $\begin{array}{r}\% \\
16.67 \\
83.33\end{array}$ \\
\hline $\begin{array}{l}\text { Anticoncepción (44) } \\
\text { No } \\
\text { Si }\end{array}$ & $\begin{array}{c}\% \\
52.27 \\
47.73\end{array}$ \\
\hline $\begin{array}{l}\text { Tabaquismo (33) } \\
\text { No } \\
\text { Si }\end{array}$ & $\begin{array}{c}\% \\
72.3(24) \\
27.27(9)\end{array}$ \\
\hline
\end{tabular}


paciente tuvo una pérdida ponderal suficiente para reclasificarse en el grupo OMS de bajo peso (Tabla 2).

En general el peso se mantuvo estable comparando el primer y último registro, con una diferencia absoluta de medias de $-0.17 \mathrm{Kg}$, sin embargo en las pacientes con metástasis, hubo una diferencia ajustada por tiempo de seguimiento de

Tabla 2. Distribución de pacientes por subgrupos de peso según clasificación de la OMS.

\begin{tabular}{|l|c|c|c|}
\hline $\begin{array}{c}\text { Grupo } \\
\text { OMS }\end{array}$ & $\begin{array}{c}\text { Inicio } \\
\text { seguimiento } \%\end{array}$ & $\begin{array}{c}\text { Final seguimiento } \\
\%\end{array}$ & $\begin{array}{c}\text { Tendencia } \\
\text { grupo }\end{array}$ \\
\hline Bajo peso & $0(0)$ & $0(0)$ & Estable \\
\hline Normopeso & $32.43(24)$ & $31.08(23)$ & Disminución \\
\hline Sobrepeso & $44.59(33)$ & $51.35(38)$ & Aumento \\
\hline Obesidad & $22.97 \%(17)$ & $17.57(13)$ & Disminución \\
\hline
\end{tabular}

$-5.3 \mathrm{Kg}(\mathrm{p}<0.05)$. Otros datos de las medidas antropométricas se presentan en la Tabla 3.

Las citas de seguimiento de las pacientes ocurrieron entre cada cuatro a seis meses. Por lo tanto, se propuso un análisis comparativo de periodos por medio de ANOVA, sin lograr significancia estadística en la comparación de medias entre pacientes con y sin recurrencia a distancia. Pero se identificó una tendencia clínica de pérdida de peso en las pacientes con recurrencia durante el tercer periodo de seguimiento (18-24 meses), respecto a sus contrapartes sin metástasis (Tabla 4).

Se consideró variabilidad ponderal clínica, si se cumplía con al menos uno de tres criterios: 1) cambio de más o menos $5 \%$ del peso inicial, 2) cambio de más o menos dos puntos IMC inicial o 3) cambio del grupo de peso OMS en cualquier momento del seguimiento (Tabla 5).

Tabla 3. Medidas antropométricas durante el seguimiento.

\begin{tabular}{|c|c|c|c|c|c|}
\hline $\begin{array}{c}\text { Medidas } \\
\text { antropométricas (74) }\end{array}$ & $\begin{array}{c}\text { Promedio } \\
\text { inicial }\end{array}$ & $\begin{array}{l}\text { Promedio } \\
\text { final }\end{array}$ & $\begin{array}{c}\text { Grupo } \\
\text { sin recurrencia }(65)\end{array}$ & $\begin{array}{c}\text { Grupo } \\
\text { con recurrencia }(9)\end{array}$ & $\mathbf{p}$ \\
\hline Talla (mt) & 1.57 & 1.57 & 1.57 & 1.57 & 0.84 \\
\hline Peso (kg) & 67.02 & 66.85 & 67.10 & 61.80 & 0.87 \\
\hline $\operatorname{IMC}\left(\mathrm{kg} / \mathrm{m}^{2}\right)$ & 26.93 & 26.88 & 27.95 & 26.77 & 0.90 \\
\hline
\end{tabular}

Tabla 4. Diferencia de promedios del peso durante los distintos momentos de medición cada cuatro a seis meses.

\begin{tabular}{|c|c|c|c|c|}
\hline $\begin{array}{c}\text { Momento } \\
\text { de seguimiento }\end{array}$ & $\begin{array}{c}\text { Promedio } \\
\text { grupo general }\end{array}$ & $\begin{array}{c}\text { Promedio } \\
\text { subgrupo sin recurrencia }\end{array}$ & $\begin{array}{c}\text { Promedio } \\
\text { subgrupo con recurrencia }\end{array}$ & ANOVA \\
\hline $\begin{array}{l}\text { Inicio del seguimiento } \\
\text { Peso inicial } \\
\text { IMC inicial }\end{array}$ & $\begin{array}{c}67.02(74) \\
26.93\end{array}$ & $\begin{array}{c}67.10(65) \\
26.95\end{array}$ & $\begin{array}{c}66.45(9) \\
26.77\end{array}$ & $\begin{array}{l}0.87 \\
0.90\end{array}$ \\
\hline $\begin{array}{l}\text { Periodo } 1 \\
\text { Peso } \\
\text { IMC }\end{array}$ & $\begin{array}{c}67.11(74) \\
26.96\end{array}$ & $\begin{array}{c}67.15(65) \\
26.95\end{array}$ & $\begin{array}{c}66.83(9) \\
26.77\end{array}$ & $\begin{array}{l}0.93 \\
0.97\end{array}$ \\
\hline $\begin{array}{l}\text { Periodo } 2 \\
\text { Peso } \\
\text { IMC }\end{array}$ & $\begin{array}{c}67.91(73) \\
27.33\end{array}$ & $\begin{array}{c}68.15(64) \\
27.43\end{array}$ & $\begin{array}{c}66.17(9) \\
26.65\end{array}$ & $\begin{array}{l}0.64 \\
0.61\end{array}$ \\
\hline $\begin{array}{l}\text { Periodo } 3 \\
\text { Peso } \\
\text { IMC }\end{array}$ & $\begin{array}{c}67.57(70) \\
27.10\end{array}$ & $\begin{array}{c}68.23(61) \\
27.36\end{array}$ & $\begin{array}{c}* 63.08(9) \\
25.37\end{array}$ & $\begin{array}{l}0.21 \\
0.15\end{array}$ \\
\hline $\begin{array}{l}\text { Periodo } 4 \\
\text { Peso } 4 \\
\text { IMC } 4\end{array}$ & $\begin{array}{c}67.03(62) \\
26.85\end{array}$ & $\begin{array}{c}67.72(53) \\
27.11\end{array}$ & $\begin{array}{c}62.95(9) \\
25.36\end{array}$ & $\begin{array}{l}0.28 \\
0.23\end{array}$ \\
\hline $\begin{array}{l}\text { Periodo } 5 \\
\text { Peso } \\
\text { IMC }\end{array}$ & $\begin{array}{l}68.24(40) \\
27.12\end{array}$ & $\begin{array}{c}68.58(34) \\
27.25\end{array}$ & $\begin{array}{c}66.3(6) \\
26.43\end{array}$ & $\begin{array}{l}0.70 \\
0.68\end{array}$ \\
\hline $\begin{array}{l}\text { Periodo } 6 \\
\text { Peso } \\
\text { IMC }\end{array}$ & $\begin{array}{c}68.12(34) \\
26.95\end{array}$ & $\begin{array}{c}68.46(28) \\
27.05\end{array}$ & $\begin{array}{c}66.55(6) \\
26.53\end{array}$ & $\begin{array}{l}0.75 \\
0.81\end{array}$ \\
\hline $\begin{array}{l}\text { Periodo } 7 \\
\text { Peso } \\
\text { IMC }\end{array}$ & $\begin{array}{c}69.52(27) \\
27.20\end{array}$ & $\begin{array}{c}71.38(22) \\
27.78\end{array}$ & $\begin{array}{c}61.34(5) \\
24.68\end{array}$ & $\begin{array}{l}0.10 \\
0.18\end{array}$ \\
\hline $\begin{array}{l}\text { Periodo } 8 \\
\text { Peso } \\
\text { IMC }\end{array}$ & $\begin{array}{c}72.85(18) \\
28.22\end{array}$ & $\begin{array}{c}73.07(16) \\
28.42\end{array}$ & $\begin{array}{c}71.05(2) \\
26.63\end{array}$ & $\begin{array}{l}0.81 \\
0.60\end{array}$ \\
\hline $\begin{array}{l}\text { Fin del seguimiento } \\
\text { Peso final } \\
\text { IMC final }\end{array}$ & $\begin{array}{c}66.85(74) \\
26.88\end{array}$ & $\begin{array}{c}67.54(65) \\
27.05\end{array}$ & $\begin{array}{c}61.88(9) \\
25.71\end{array}$ & $\begin{array}{l}0.18 \\
0.40\end{array}$ \\
\hline
\end{tabular}


Tabla 5. Frecuencia de la variabilidad del peso de acuerdo con las distintas definiciones aceptadas en el estudio.

\begin{tabular}{|c|c|c|c|c|}
\hline Criterio de variabilidad & General (74) \% & Sin recurrencia $(65) \%$ & Con recurrencia (9) \% & Valor de $\mathbf{p}$ \\
\hline $\begin{array}{l}5 \% \text { peso inicial } \\
\text { Ausente } \\
\text { Presente }\end{array}$ & $\begin{array}{l}40.54(30) \\
59.45(44)\end{array}$ & $\begin{array}{l}33.78 \\
54.05\end{array}$ & $\begin{array}{l}6.75 \\
5.40\end{array}$ & 0.32 \\
\hline $\begin{array}{l}2 \text { puntos del IMC } \\
\text { Ausente } \\
\text { Presente }\end{array}$ & $\begin{array}{l}55.41(41) \\
44.59(33)\end{array}$ & $\begin{array}{l}47.29 \\
40.54\end{array}$ & $\begin{array}{l}8.10 \\
4.05\end{array}$ & 0.46 \\
\hline $\begin{array}{c}\text { Grupo OMS } \\
\text { Ausente } \\
\text { Presente }\end{array}$ & $\begin{array}{l}75.68(56) \\
24.34(18)\end{array}$ & $\begin{array}{l}68.91 \\
18.91\end{array}$ & $\begin{array}{l}6.75 \\
5.40\end{array}$ & 0.13 \\
\hline $\begin{array}{l}\text { Al menos un criterio } \\
\text { Ausente } \\
\text { Presente }\end{array}$ & $\begin{array}{l}33.78(25) \\
66.22(49)\end{array}$ & $\begin{array}{l}29.72 \\
58.10\end{array}$ & $\begin{array}{l}4.05 \\
8.10\end{array}$ & 0.97 \\
\hline Diferencia absoluta del peso & $-0.17 \mathrm{~kg}$ & $+0.50 \mathrm{~kg}$ & $-5.06 \mathrm{Kg}$ & 0.01 \\
\hline
\end{tabular}

\section{Discusión}

A la fecha este es el primer estudio en población colombiana con un diseño longitudinal, interesado en describir el comportamiento del peso en mujeres con cáncer de mama. La razón de escoger solo a pacientes del tipo luminal A, se debió a que se reconoce que los tumores con receptores hormonales son más susceptibles a la producción extraovárica de estrógenos $(7,8)$ y parte de la heterogeneidad en los resultados de muchos estudios, se debe a no reconocer esta susceptibilidad diferencial entre subtipos intrínsecos.

Datos poblacionales en Colombia (ENSIN 2010) similares a como se registra en países desarrollados, muestran una alarmante prevalencia de sobrepeso/obesidad principalmente en mujeres, llegando hasta más de $60 \%$ de las mujeres a partir de los 40 años (9).

En este estudio se encontró una prevalencia de sobrepeso/ obesidad de $67.56 \%$ desde el inicio del seguimiento y se mantuvo prácticamente sin cambios a pesar del tratamiento multimodal $(68.92 \%)$. Llamativamente ninguna mujer presentó bajo peso al principio ni al final del estudio. Lo anterior plantea que en esta población el exceso de peso puede ser una característica común entre las mujeres con diagnóstico de cáncer de mama como se ha descrito en otros estudios (10).

La evidencia sugiere que la relación del exceso de peso con el riesgo de cáncer de mama es diferencial entre mujeres pre y posmenopáusicas. Hay datos que proponen una relación inversa o protectora del sobrepeso en mujeres jóvenes antes de la menopausia (11). No obstante estos resultados contradictorios pueden explicarse por una mayor presentación de tumores de tipo basal en mujeres jóvenes, cuya susceptibilidad biológica a los efectos de la masa grasa no están bien comprendidos. Nuevamente este estudio supera dicha dificultad homogenizando a las pacientes por subtipo molecular y esto podría explicar que, a pesar de que $51 \%$ de las mujeres de esta cohorte eran menores de 50 años y la mayoría premenopáusicas, no tuvieron ninguna diferencias estadística en las variables de interés comparadas con las pacientes que reportaron ser menopáusicas. Tal vez el efecto del peso en mujeres con cáncer de mama luminal A es independiente del estado de fertilidad de las pacientes.

Es importante comparar los resultados reportados por Cuello-López y Cols, que presentaron un estudio que analizó el peso como variable de pronóstico en Colombianas, valiéndose de un diseño de corte transversal en 2017 (12), que mostró que pacientes posmenopáusicas de un centro oncológico en Medellín presentaron una asociación positiva entre IMC y subtipo luminal A. En el mismo trabajo se encontró una baja prevalencia $(1.53 \%)$ de mujeres con cáncer de mama con bajo peso, mientras que hubo una alta frecuencia $(65.4 \%)$ de sobrepeso/obesidad, similar a lo descrito en nuestra investigación. Por su parte Gutiérrez y colaboradores describieron un grupo de 45 pacientes en México (13) todas en estadio localmente avanzado (III) con una alta prevalencia $(86.6 \%)$ de sobrepeso/obesidad, dato concordante con nuestros hallazgos y con otros estudios en el hemisferio con altas prevalencias de sobrepeso reportadas constantemente mayores a $50 \%$ en población con características similares a las de las pacientes presentadas en este trabajo.

Una de las fortalezas de este estudio fue analizar el comportamiento temporal del peso valorando su tendencia durante varios momentos de observación de las pacientes, por tal razón uno de los principales datos de interés fue la variabilidad ponderal definida por varios criterios (IMC, peso en $\mathrm{Kg}$ y grupo OMS). En la literatura se han identificado patrones de comportamiento ponderal de acuerdo con el índice de masa corporal antes del tratamiento. Así, las mujeres con IMC más cercano al normal presentaron una mayor tendencia a ganar peso en comparación de las obesas que incluso tienden a bajar (14). En esta cohorte resalta la estabilidad ponderal entre el primer y último registro, pero hay que advertir que sí hubo variabilidad en el peso entre dichos periodos pero la tendencia fue a estabilizarse volviendo al peso inicial. 
A pesar de que existen estudios con muestras poblacionales importantes, como el de Chen y cols. en China que reportan una tendencia general de ganancia de peso cercana a dos $\mathrm{Kg}$ en los primeros 18 meses después de quimioterapia (15). En este trabajo se encontró una tendencia sutil pero global a la pérdida de peso $(-0.17 \mathrm{~kg})$ que se aleja del comportamiento descrito y también en estudios occidentales como el Women's Healthy Eating and Living Study (WHEL). Nuevamente podría tratarse de diferencias étnicas, lo que justifica la realización de este tipo de estudios para registrar las diferencias poblacionales.

La tasa de recurrencia fue $12,1 \%$ que es significativamente mayor a lo reportado en otros estudios, con riesgo de metástasis menor a $9 \%$ durante periodos de observación más extensos (60 meses) $(16,17)$. El diseño de este trabajo no identifica las posibles causas asociadas al relativo aumento en el número de eventos de metástasis. Sin embargo, se reconoce que en pacientes con sobrepeso/obesidad como en la mayoría de las participantes en esta cohorte, se presentan peores desenlaces $(8,18,19)$. Por ejemplo las neoplasias en mujeres con sobrepeso tienen mayor tamaño, peor índice de proliferación y más número de metástasis linfáticas (20).

Se debe resaltar que hubo diferencia estadística en la cantidad de pérdida de peso entre pacientes con y sin recurrencia a distancia, un fenómeno que ocurrió incluso antes del diagnóstico clínico de metástasis. En la mayoría de los casos la pérdida de peso fue clínicamente significativa a partir de los primeros 18 meses del seguimiento. Esto da vigencia a la valoración longitudinal del peso en pacientes oncológicas como un indicador clásico de neoplasia activa.

Para terminar se debe reconocer que se trata de un estudio limitado por el pequeño tamaño de muestra, corto periodo de seguimiento, ausencia de otras mediciones antropométricas más específicas y falta de aleatorización. Se espera que este trabajo siente las bases para realizar estudios prospectivos y analíticos interesados en seguir analizando la relación entre peso y cáncer en población latinoamericana, con una metodología más robusta que permita mejorar el poder y el alcance de los resultados.

\section{Conclusiones}

El sobrepeso y obesidad son una característica muy prevalente en pacientes con cáncer de mama luminal A en estadio localmente avanzado. No hubo evidencia concluyente de aumento de riesgo de metástasis o muerte asociado a exceso de peso en esta población, al contrario la pérdida de peso fue una característica estadísticamente significativa de pacientes que presentaron recurrencia a distancia durante el seguimiento, sin poderse definir como un factor causal.

\section{Referencias}

1. Kroenke, C. H., Chen, W. Y., Rosner, B., \& Holmes MD. Weight, weight gain, and survival after breast cancer diagnosis. J Clin Oncol. 2005; 23(7): 1370-1378.
2. Hursting SD, Berger NA. Energy Balance, Host-Related Factors, and Cancer Progression. J Clin Oncol [Internet]. 2010 [cited 2019 Oct 27];28:4058-65. Available from: www.jco.org

3. Majed B, Moreau T ABCIBCG. Overweight, obesity and breast cancer prognosis: optimal body size indicator cut-points. Breast Cancer Res Treat. 2009;115(1):193-203.

4. Dignam JJ, Wieand K, Johnson KA, Raich P, Anderson SJ, Somkin C, et al. Effects of obesity and race on prognosis in lymph node-negative, estrogen receptor-negative breast cancer. Breast Cancer Res Treat [Internet]. 2006 Jun 6 [cited 2019 Oct 27]; 97(3): 245-54. Available from: http://www.ncbi.nlm.nih. gov/pubmed/16331345

5. de Azambuja E, McCaskill-Stevens W, Francis P, Quinaux E, Crown JPA, Vicente M, et al. The effect of body mass index on overall and disease-free survival in node-positive breast cancer patients treated with docetaxel and doxorubicincontaining adjuvant chemotherapy: the experience of the BIG 02-98 trial. Breast Cancer Res Treat [Internet]. 2010 Jan 3 [cited 2019 Oct 27];119(1):145-53. Available from: http://www.ncbi.nlm.nih.gov/pubmed/19731015

6. Moon H-G, Han W, Noh D-Y. Underweight and Breast Cancer Recurrence and Death: A Report From the Korean Breast Cancer Society. J Clin Oncol [Internet]. 2009 Dec 10 [cited 2019 Oct 27];27(35):5899-905. Available from: http://www. ncbi.nlm.nih.gov/pubmed/19805676

7. Yang XR, Chang-Claude J, Goode EL, Couch FJ, Nevanlinna H, Milne RL, et al. Associations of Breast Cancer Risk Factors With Tumor Subtypes: A Pooled Analysis From the Breast Cancer Association Consortium Studies. Artic I JNCI [Internet]. 2011 [cited 2019 Oct 13];103:250. Available from: https://academic. oup.com/jnci/article-abstract/103/3/250/2517238

8. Ligibel JA, Strickler HD. Obesity and Its Impact on Breast Cancer: Tumor Incidence, Recurrence, Survival, and Possible Interventions [Internet]. 2013 [cited 2019 Sep 15]. Available from: https://media4.asco.org/132/edbook/pdfs/ EdBookAM20133352.pdf

9. Herrán ÓF, Patiño GA, Del Castillo SE. La transición alimentaria y el exceso de peso en adultos evaluados con base en la encuesta de la situación nutricional en Colombia, 2010. Biomedica. 2016 Jan 1;36(1):109-20.

10. Pacholczak R, Klimek-Piotrowska W, Kuszmiersz P. Associations of anthropometric measures on breast cancer risk in pre-and postmenopausal women-a case-control study. 2016 [cited 2019 Oct 14]; Available from: https://www.ncbi. nlm.nih.gov/pmc/articles/PMC4782382/pdf/40101_2016_Article_90.pdf

11. Calle EE, Thun MJ. Obesity and cancer. Oncogene [Internet]. 2004 [cited 2019 Sep 15];23:6365-78. Available from: http://www.cdc.gov/nchs/nhanes.htm

12. Cuello-López J, Fidalgo-Zapata A, Vásquez-Trespalacios E. Obesity and Prognostic Variables in Colombian Breast Cancer Patients: A Cross-Sectional Study. 2017 [cited 2019 Sep 15]; Available from: https://doi.org/10.1155/2017/9574874

13. Gutiérrez G, Autrique M, Ceballos G. Sobrepeso y obesidad: frecuentes características del estado nutricional en una muestra de pacientes mexicanas con cáncer de mama localmente avanzado. Rev Colomb Cancerol. 2012; 16(3): 187-91.

14. Makari-Judson G. Weight gain following breast cancer diagnosis: Implication and proposed mechanisms. World J Clin Oncol. 2014;

15. Chen X, Gu K, Lu W, Zheng Y, Chen Z, Shu XO, et al. Weight Change and Its Correlates Among Breast Cancer Survivors. Nutr Cancer. 2011;

16. Lord SJ, Kiely BE, Pearson SA, Daniels B, O'Connell DL, Beith J, et al. Metastatic breast cancer incidence, site and survival in Australia, 2001-2016: A population-based health record linkage study protocol. Vol. 9, BMJ Open. BMJ Publishing Group; 2019.

17. Yadav BS, Sharma SC, Singh R, Singh G. Patterns of relapse in locally advanced breast cancer treated with neoadjuvant chemotherapy followed by surgery and radiotherapy. J Cancer Res Ther [Internet]. 2007;3(2):75-80. Available from: http://www.ncbi.nlm.nih.gov/pubmed/17998727

18. Bandera E V, Maskarinec G, Romieu I, John EM. Racial and Ethnic Disparities in the Impact of Obesity on Breast Cancer Risk and Survival: A Global Perspective 1-3. [cited 2019 Sep 15]; Available from: https://academic.oup.com/advances/ article-abstract/6/6/803/4555151

19. Del Fabbro E, Parsons H, Warneke CL, Pulivarthi K, Litton JK, Dev R, et al. The Relationship Between Body Composition and Response to Neoadjuvant Chemotherapy in Women with Operable Breast Cancer. Oncologist. 2012 Oct 1;17(10):1240-5.

20.Payandeh M, Sadeghi M, Sadeghi E. Differences in Prognostic Factors between Early and Late Recurrence Breast Cancers. Asian Pac J Cancer Prev [Internet]. 2015;16(15):6575-9. Available from: http://www.ncbi.nlm.nih.gov/ pubmed/26434877 